\title{
The Impact of Two Semiannual Treatments with Albendazole Alone on Lymphatic Filariasis and Soil-Transmitted Helminth Infections: A Community-Based Study in the Republic of Congo
}

\author{
Sébastien D. S. Pion,* Cédric B. Chesnais, Jean Bopda, Frédéric Louya, Peter U. Fischer, Andrew C. Majewski, \\ Gary J. Weil, Michel Boussinesq, $\dagger$ and François Missamou $\dagger$ \\ Unité Mixte Internationale 233, Institut de Recherche pour le Développement and University of Montpellier 1, Montpellier, France; \\ Centre for Research on Filariasis and Other Tropical Diseases (CRFilMT), Yaoundé, Cameroon; Programme National de Lutte contre \\ l'Onchocercose, Ministère de la Santé et de la Population, Brazzaville, Republic of Congo; Infectious Diseases Division, \\ Department of Internal Medicine, Washington University School of Medicine, St. Louis, Missouri
}

\begin{abstract}
Implementation of mass drug administration (MDA) with ivermectin plus albendazole (ALB) for lymphatic filariasis (LF) has been delayed in central Africa because of the risk of serious adverse events in subjects with high Loa loa microfilaremia. We conducted a community trial to assess the impact of semiannual MDA with ALB (400 mg) alone on LF and soil-transmitted helminth (STH) infections in the Republic of Congo. Evaluation at 12 months showed that ALB MDA had not significantly reduced Wuchereria bancrofti antigenemia or microfilaria (mf) rates in the community (from $17.3 \%$ to $16.6 \%$ and from $5.3 \%$ to $4.2 \%$, respectively). However, the geometric mean mf count in mf-positive subjects was reduced from 202.2 to $80.9 \mathrm{mf} / \mathrm{mL}(60 \%$ reduction, $P=0.01)$. The effect of ALB was impressive in 38 subjects who were mf-positive at baseline and retested at 12 months: $37 \%$ had total mf clearance, and individual mf densities were reduced by $73.0 \%$. MDA also dramatically reduced the hookworm infection rate in the community from $6.5 \%$ to $0.6 \%$ (91\% reduction), with less impressive effects on Ascaris and Trichuris. These preliminary results suggest that semiannual community MDA with ALB is a promising strategy for controlling LF and STH in areas with coendemic loiasis.
\end{abstract}

\section{INTRODUCTION}

Lymphatic filariasis (LF) is a severely debilitating and disabling parasitic disease that is endemic in 73 tropical and subtropical countries. An estimated 1.4 billion individuals live in endemic areas. LF in Africa is caused by Wuchereria bancrofti and transmitted mainly by Anopheles or Culex mosquitoes. In 2000, the World Health Organization (WHO) launched an elimination program for LF that was based on an innovative rapid mapping procedure to identify endemic areas and annual mass drug administration (MDA) of albendazole (ALB; $400 \mathrm{mg}$ ) plus ivermectin (IVM; 150$200 \mu \mathrm{g} / \mathrm{kg}$ ) in countries where onchocerciasis is coendemic and ALB plus diethylcarbamazine (DEC) in other countries. More than 4 billion treatments were delivered to populations in need between 2000 and 2012, and this has had a substantial impact on infection rates and intensity of transmission. ${ }^{1,2}$ However, the presence of Loa loa in central Africa prevents the use of either IVM or DEC in LF elimination programs in that region, because these drugs sometimes cause fatal adverse events in individuals with high L. loa microfilaremia. ${ }^{3-5}$ This situation has jeopardized the goal of eliminating LF by $2020 .^{6,7}$

MDA using ALB alone may be a suitable strategy for LF elimination in loiasis-endemic areas. ALB is safe for use in persons with loiasis. ${ }^{8}$ In addition, several clinical trials showed that a single dose of ALB can reduce $W$. bancrofti microfilaria (mf) densities by $50 \%$ or more, and this reduction is sustained for 1 year or more after treatment. Of course, the decline in mf counts after ALB alone was slower and less dramatic than after combination treatment with either IVM + ALB or DEC $+\mathrm{ALB},{ }^{9,10}$ but a small study found that a second dose of

*Address correspondence to Sébastien D. S. Pion, Institut de Recherche pour le Développement (UMI 233), 911 Avenue Agropolis, BP 64501, 34394 Montpellier Cedex 5, France. E-mail: sebastien.pion@ird.fr

$\dagger$ These authors contributed equally.
ALB given 1 year after the first led to a further decrease in mf counts. ${ }^{11}$

The aim of MDA in LF elimination programs is to reduce the $\mathrm{mf}$ reservoir in populations to levels that cannot support sustained transmission of $W$. bancrofti by mosquitoes. It is estimated that four to six annual rounds of MDA with ALB + IVM or ALB + DEC are needed to interrupt transmission. ${ }^{12-14}$ Clinical trials also showed that semiannual treatment with DEC alone, ${ }^{15-17}$ IVM alone, ${ }^{16,17}$ or a combination of DEC + IVM ${ }^{18,19}$ is more effective for reducing mf densities than annual treatment. In 2012, the WHO proposed a provisional strategy for controlling LF in areas with coendemic loiasis where onchocerciasis is absent. ${ }^{1}$ This includes MDA with ALB (preferably semiannually) together with integrated vector management. However, the evidence base for this policy is thin, and more information is needed on the efficacy of MDA with ALB alone for LF control or elimination in community settings. Shortly before the new WHO proposition was announced, we independently started a 3-year community trial of semiannual ALB MDA for LF in an endemic village in the Republic of Congo. This paper reports the impact of two rounds of MDA with ALB on LF and soiltransmitted helminthiases (STHs) in this village.

\section{MATERIALS AND METHODS}

Study design. The objective of this study is to assess the impact of semiannual community treatments with ALB on LF and STH over a 3-year period. Here, we present results that show the impact of two rounds of ALB. The study is conducted in Seke Pembe village, which is located in a savanna area of the Bouenza division in the Republic of Congo. Baseline assessments for LF and STH were conducted between September 18, 2012 and October 8, 2012 (period referred as October of 2012 below). Community MDA with ALB was provided just after the parasitological examinations, and a second treatment was given in early April of 2013. The assessment survey was 
conducted between September 30, 2013 and October 12, 2012 (period referred as October of 2013 below; i.e., 6 months after the second round of MDA).

An exhaustive census of the population living in the study village was conducted in August of 2012, approximately 1 month before the start of the trial. The census was updated before the second round of MDA so that we could compute precise figures for therapeutic coverage and ascertain the treatment history for each individual. ALB was offered to all individuals aged 2 years old or older, but parasitological assessments were only performed for those $\geq 5$ years of age.

Detection of $\boldsymbol{W}$. bancrofti infections. $W$. bancrofti infections were detected by antigen testing and microscopy. Circulating filarial antigens (CFAs) were detected with the Binax Filariasis Now card test (ICT; Alere, Scarborough, ME) according to the manufacturer's instructions. ICT cards were read at 10 minutes by a single trained operator as previously described. $^{20}$ The results were scored semiquantitatively as follows: negative tests with no visible test $(\mathrm{T})$ line were assigned a score of 0 , tests with a clearly visible $\mathrm{T}$ line that was weaker than the control $\mathrm{C}$ line were assigned a score of 1, tests with a $\mathrm{T}$ line approximately as dark as the control line were scored as 2 , and cards with a $\mathrm{T}$ line darker than the $\mathrm{C}$ line were scored as 3 . All individuals with a positive ICT test (score $\geq 1$ ) were invited to return for a second blood sampling between 10:00 PM and 1:00 AM for assessment of $W$. bancrofti mf. Fingers were cleaned with an alcohol wipe and pricked with a $1.5 \times 2.0 \mathrm{~mm}$ BD Microtainer contact-activated lancet (Becton Dickenson, Franklin Lakes, NJ). Blood was collected with a capillary tube, and two blood smears (volume of $70 \mu \mathrm{L}$ each) were prepared for each subject. On the next day, the blood smears were dehemoglobinized, stained with Giemsa, and examined by two experienced microscopists. Each microscopist read one of two slides from each subject. Because loiasis and Mansonella perstans filariasis are also endemic in some parts of the Bouenza division, special attention was paid to identify the species of each $\mathrm{mf}$ present in the thick smears, and counts were recorded separately for each species identified. The mf count for individual subjects was defined as the arithmetic mean (AM) of the counts made on the two slides expressed as mf per milliliter of blood.

Detection of STH infections. Every day, about 10 households listed in the census register were visited, and all occupants aged 5 years old or older were asked to report for testing and treatment on the next day. They were also given a $50 \mathrm{~mL}$ plastic stool container and asked to collect a sample of their stool during the early hours of the following morning. The stools specimens were collected every day at around 9 AM, stored in cooling boxes with ice packs, and shipped within 6 hours to the laboratory, where they were placed in a refrigerator at $6^{\circ} \mathrm{C}$. From each sample, two thick smears were prepared within 24 hours of collection according to the modified Kato-Katz method. Slides were examined by microscopy at $\times 40$ magnification within 1 hour after preparation, and results were expressed as eggs per gram of stool (epg).

Drug distribution and therapeutic coverage. Individuals who had a negative ICT result were immediately treated with ALB (400 mg), which was taken on the spot under direct observation of investigators. Those with positive ICT test results received the drug just after collection of night blood. Finally, all inhabitants who had not participated in the parasitological survey were visited at home and offered ALB treat- ment. All drugs were distributed under the supervision of a local healthcare worker who was also in charge of the population census. For treatment of young children, ALB tablets were crushed with a spoon and mixed with water. Although the WHO considers that pregnant women can be treated with ALB during the second or third trimester, we decided to exclude all pregnant women from MDA. Nonetheless, ALB was offered to them by the local healthcare worker after they gave birth.

The therapeutic coverage was calculated as the number of individuals who received the drug divided by the eligible population ( $\geq 2$ years of age) recorded during the census that was conducted just before the MDA.

Statistical analysis. Changes in infection rates from the baseline examination to year 1 were analyzed using comparisons of both cross-sectional (all individuals present at the time of observation) and longitudinal (limited to individuals examined at both baseline and 12 months after the first treatment) data.

In the cross-sectional analyses, infection rates at baseline and 12 months after the first treatment were compared for each parasite with the $\chi^{2}$ test, and infection intensities (mf per milliliter for LF or epg for STH) were compared using the Mann-Whitney test. Prevalence rates and infection intensities in the cohort of individuals examined at both time points were performed using McNemar and Wilcoxon signed ranks tests, respectively. Mean infection intensity measures for W. bancrofti (mf per milliliter) and STH (epg) are reported as AMs and geometric means (GMs), respectively. The latter was computed as the Williams' mean $\left(e^{\frac{1}{N} \sum_{1}^{N} X+1}-1\right)$, where $X$ is the individual $\mathrm{mf}$ or egg density, and $N$ is the number of examined individuals. This assumes that persons with negative ICT test results had mf counts of zero. For STH infections, we also considered classes of infection intensity in accordance with the WHO guidelines. ${ }^{21}$

Ethical clearance. This study was approved by the Ethics Committee for Research in Health Sciences of the Republic of Congo and conducted with personnel of the Ministry of Health and Population of the Republic of Congo. The purposes of the study were presented during meetings with village leaders and then explained in French and in the local language (Kituba) to all participants both orally and in a written document that was given to each individual. Adult participants signed an informed consent form. Participants younger than 18 years of age were enrolled only if they expressed verbal assent to participate in the study and at least one parent signed a consent form.

\section{RESULTS}

Study population. In August of 2012, Seke Pembe had a population of 1,055 inhabitants, among which 876 were $\geq 5$ years of age. In October of 2012, 773 were tested for LF, with a sex ratio (male:female) of $1: 1.2$ and median age of 23 years old (interquartile range $[\mathrm{IQR}]=10-40$ years old). In October of 2013, 1,010 individuals were recorded at the census (including 853 aged 5 years old and over), and 741 were examined for $\mathrm{LF}$ ( $\mathrm{IQR}=10-41$ years old). Five hundred eighty-eight subjects were tested in both years.

Among 185 individuals lost to follow-up between October of 2012 and October 2013, 5 were deceased, 14 refused to be 
TABLE 1

Impact of two semiannual community treatments with ALB on $W$. bancrofti infection (cross-sectional study)

\begin{tabular}{|c|c|c|c|c|}
\hline & $2012(N=773)$ & $2013(N=741)$ & Reduction (\%) & $P$ value \\
\hline Prevalence of CFA (\%) & $17.3[14.7-20.0]^{*}$ & $16.6[13.9-19.3]$ & 4.3 & 0.703 \\
\hline Prevalence of $\mathrm{mf}(\%)$ & $5.3[3.7-6.9]$ & $4.2[2.7-5.6]$ & 21.1 & 0.309 \\
\hline GM of $\mathrm{mf}$ densities in the community $(\mathrm{mf} / \mathrm{mL})$ & $0.32[0.21-0.45]$ & $0.20[0.12-0.29]$ & 37.5 & 0.273 \\
\hline $\mathrm{GM}$ of $\mathrm{mf}$ densities in microfilaremic individuals $(\mathrm{mf} / \mathrm{mL})$ & $202.2[122.8-332.6]$ & $80.9[46.9-139.1]$ & 60.0 & 0.010 \\
\hline AM of $\mathrm{mf}$ densities in microfilaremic individuals $(\mathrm{mf} / \mathrm{mL})$ & $525.4[276.6-774.3]$ & $217.8[88.0-347.5]$ & 58.6 & 0.010 \\
\hline
\end{tabular}

This table summarizes results from two surveys performed in 2012 and 2013.

*Numbers in brackets indicate $95 \%$ CIs.

examined again, 53 were absent from the village during the 2013 survey, and 113 had moved away from the village.

Therapeutic coverage. In October of 2012, 985 individuals age $\geq 2$ years old were eligible for treatment with ALB, and $871(88.4 \%)$ received the treatment. Six months later, in March of 2013, the total population was 1,032, including 967 individuals aged $\geq 2$ years old, and $825(85.3 \%)$ were treated with ALB. Among 588 individuals who were examined for LF in both October of 2012 and 1 year later, 492 (83.7\%) had received two treatments with ALB, and 94 (16.0\%) had received only one of two treatments ( 87 were treated only in October of 2012 and 7 were treated only in April of 2013). Two subjects $(0.3 \%)$ were examined two times but did not receive the treatment.

Among 153 newly examined individuals in October of 2013, a history of ALB treatment was obtained for 148; 73 (49.3\%), $35(23.6 \%)$, and 40 (27\%) individuals had received zero, one, and two ALB treatments, respectively, as part of our study.

Impact of two semiannual community ALB treatments on W. bancrofti. Considering cross-sectional data from the total population studies, no significant decrease in LF prevalence was observed between 2012 and 2013. The CFA rate decreased from $17.3 \%$ to $16.6 \%(P=0.703)$, and the mf rate decreased from $5.3 \%$ to $4.2 \%(P=0.309)$ (Table 1$)$. The GM of $\mathrm{mf}$ densities in the community decreased by $37.5 \%(P=$ 0.273 ). Nevertheless, $\mathrm{mf}$ densities among microfilaremic individuals decreased significantly between the two rounds, with GM and AM of mf densities decreasing by $60 \%$ and $58.6 \%$, respectively $(P=0.010)$ (Table 1$)$. No L. loa or $M$. perstans $\mathrm{mf}$ were detected during the survey.

Among 588 individuals examined in both 2012 and 2013, there were significant decreases in CFA prevalence, mf prevalence, and infection intensity (Table 2). CFA prevalence decreased from $19.7 \%$ to $17.9 \%(P<0.001)$, and mf prevalence decreased from $6.5 \%$ to $4.3 \%(P<0.001)$, which correspond to $9.5 \%$ and $34.3 \%$ reduction, respectively. The GMs of $\mathrm{mf}$ densities in 38 individuals presenting with $W$. bancrofti $\mathrm{mf}$ at baseline dramatically decreased from 200.4 to $13.2 \mathrm{mf} / \mathrm{mL}$ in 2013 (93.4\% reduction, $P$ value $<0.001)$ (Figure 1 and Table 2). Fourteen of these subjects $(36.8 \%)$ had no $\mathrm{mf}$ detected in night blood in 2013. The mean percentage reduction in $\mathrm{mf}$ counts in these 38 individuals was $73.0 \%$ (95\% confidence inter- val $[95 \% \mathrm{CI}]=57.3-88.5)$. The mean percentage reduction in $\mathrm{mf}$ counts for 30 subjects with more than $100 \mathrm{mf} / \mathrm{mL}$ at baseline was $79.1 \%(95 \% \mathrm{CI}=69.6-88.7)$, and 8 of these 30 individuals (26.7\%) were amicrofilaremic in 2013.

Changes in the ICT scores between October of 2012 and October of 2013 are presented in Table 3 for people tested at both time points. Scores decreased in 44 of 116 people $(37.9 \%)$ with positive tests at baseline, including 15 persons $(12.9 \%)$ for whom ICT card tests were negative in 2013. However, 3 of those 116 persons $(2.6 \%)$ had increased scores 1 year later. In addition, 4 of 472 individuals $(0.8 \%)$ with negative ICT tests in 2012 were ICT-positive in 2013. This corresponds to an incidence rate for filarial antigenemia of 8.5/1,000 person-years.

Impact of two semiannual community ALB treatments on STH infections. In total, 462 subjects (199 males and 263 females, median age $=22$ years old, range $=5-92$ years old) provided stool samples that were examined for STH by KatoKatz during the baseline survey in 2012, and 335 subjects (160 males and 175 females, median age $=26$ years old, range $=$ 5-75 years old) were tested by Kato-Katz in 2013; 162 individuals had stool testing at both time points. STH results are summarized in Tables 4-6.

The prevalence of Ascaris lumbricoides infection in the surveyed samples (cross-sectional data) decreased from $56.4 \%$ in October of 2012 to $36.4 \%$ in October of 2013 (35.5\% reduction; $P<0.001)$. Infection intensities were significantly reduced in children but not in adults 6 months after two rounds of MDA (Table 4). Nonetheless, a clear shift of distribution toward lower classes of intensity was observed, and no subjects were found in the highest intensity class after two rounds of MDA $\left(\chi^{2}=41.26, P=0.002\right)$ (Figure 2). Among 162 individuals reexamined after two rounds of MDA, 103 (63.6\%) were initially positive for $A$. lumbricoides eggs; 61 (59.2\%) of those 103 individuals had negative stool tests for Ascaris in October of 2013 (6 months after the second round of ALB).

The baseline rate for Trichuris trichiura infection was $78.6 \%$ ( $92.2 \%$ in children $5-14$ years of age); this rate decreased slightly to $71.0 \%$ in October of 2013 (9.8\% reduction, $P=$ 0.015) (Table 5). T. trichiura infection intensity in infected persons decreased from 459.1 to 233.9 epg (49.1\% reduction, $P<0.001)$, with a shift toward lower infection classes after $\operatorname{ALB}\left(\chi^{2}=16.62, P<0.0001\right)$ (Figure 3$) ; 33$ of 131 individuals

TABLE 2

Effect of two semiannual treatments with ALB on W. bancrofti infection (longitudinal study)

\begin{tabular}{|c|c|c|c|c|c|}
\hline & $N$ & 2012 & 2013 & Reduction (\%) & $P$ value \\
\hline Prevalence of CFAs (\%) & 588 & $19.7[16.5-23.0]^{*}$ & $17.9[14.8-21.0]$ & 9.5 & 0.019 \\
\hline Prevalence of $\mathrm{mf}(\%)$ & 588 & $6.5[4.5-8.5]$ & $4.3[2.6-5.9]$ & 34.3 & 0.001 \\
\hline $\mathrm{GM} \mathrm{mf}$ density in persons with $\mathrm{mf}$ at baseline $(\mathrm{mf} / \mathrm{mL})$ & 38 & $200.4[120.6-332.5]$ & $13.2[5.8-28.6]$ & 93.4 & $<0.001$ \\
\hline AM mf density in persons with $\mathrm{mf}$ at baseline $(\mathrm{mf} / \mathrm{mL})$ & 38 & $490.1[250.2-729.9]$ & $77.1[33.3-120.9]$ & 84.3 & $<0.001$ \\
\hline
\end{tabular}

This table summarizes W. bancrofti infection parameters in the subset of the village population that was studied in both 2012 and 2013 ( $N=588$ ). GMs and AMs in 2013 include negative subjects.

* Numbers in brackets indicate $95 \%$ CIs. 


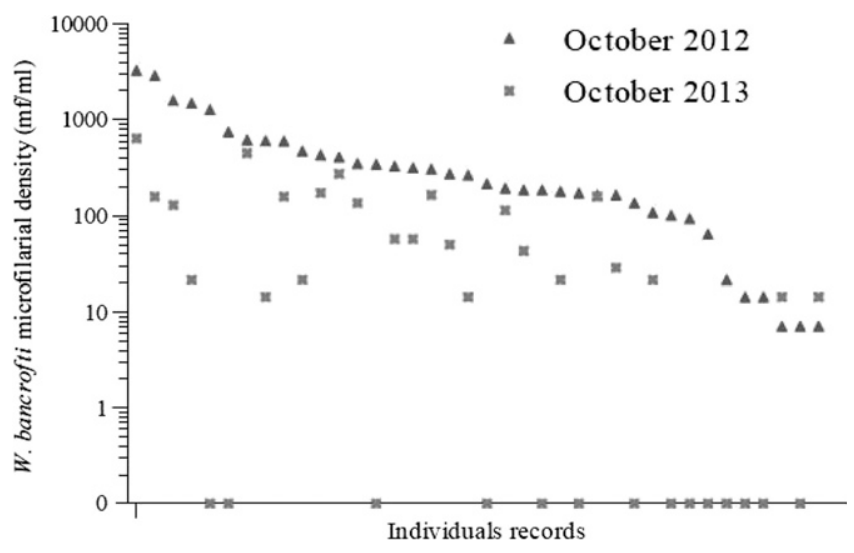

Figure 1. W. bancrofti $\mathrm{mf}$ densities before and 6 months after two semiannual treatments with ALB. This graph shows data from 38 individuals who were microfilaremic at baseline (ranked by decreasing mf density in 2012) and retested 12-13 months later. Note that a $\log$ scale was used for mf density.

$(25.2 \%)$ who were positive for Trichuris at baseline were negative in October of 2013.

The baseline rate for hookworm infection was $6.5 \%(7.8 \%$ in adults and $4.4 \%$ in children) (Table 6), and intensities were low. No children were positive for hookworm in October of 2013 (6 months after the second round of ALB), and hookworm prevalence in adults was $0.9 \%$ at that time $(88.1 \%$ reduction, $P<0.001)$. Hookworm infection intensity in positive stool samples decreased from 50.1 to 17 epg $(66.1 \%$ reduction) in adults, but this difference was not statistically significant $(P=0.072)$.

\section{DISCUSSION}

In 2005, a Cochrane review of ALB trials for LF concluded that ALB alone was not better than placebo for clearing blood mf, but this was based on results from single-dose treatment trials. ${ }^{22}$ The WHO recently proposed that, in those areas where LF is coendemic with L. loa and onchocerciasis is not meso- or hyperendemic, LF control programs use MDA (semiannual preferred) with ALB alone in association with integrated vector control. ${ }^{1}$ This study showed that mf counts and filarial antigen test scores in infected individuals were impressively reduced 6 months after two semiannual rounds of ALB, although population prevalence rates of $\mathrm{mf}$ and antigenemia were not changed. These preliminary results from the first community trial of ALB MDA for LF support the WHO's provisional strategy.

It is interesting to compare results from Seke Pembe with those obtained in areas where the standard annual MDA

TABLE 3

Evolution of filarial antigenemia (ICT) scores in 588 individuals tested in 2012 and in 2013.

\begin{tabular}{crrrrr}
\hline & \multicolumn{5}{c}{ ICT score in October of 2013 } \\
\cline { 2 - 6 } ICT score in October of 2012 & \multicolumn{1}{c}{0} & \multicolumn{1}{c}{1} & 2 & 3 & Total \\
\hline 0 & 468 & 4 & 0 & 0 & 472 \\
1 & 15 & 54 & 1 & 0 & 70 \\
2 & 0 & 19 & 13 & 2 & 34 \\
3 & 0 & 0 & 10 & 2 & 12 \\
Total & 483 & 77 & 24 & 4 & 588 \\
\hline
\end{tabular}

regimen recommended for most areas in sub-Saharan Africa had been applied (annual IVM + ALB). A clinical trial in Ghana reported that $W$. bancrofti $\mathrm{mf}$ prevalence and intensity were reduced by about $23 \%$ and $87 \%$ from baseline values, ${ }^{23}$ respectively, 1 year after a single dose of IVM + ALB. Results from this study (with a $34.3 \%$ reduction in mf prevalence and an approximately $90 \%$ reduction in $\mathrm{mf}$ counts at 1 year) in reexamined individuals suggest that semiannual ALB is as effective as annual IVM + ALB. The Ghana study also reported that $\mathrm{mf}$ prevalence and $\mathrm{mf}$ counts decreased by $16.1 \%$ and $88.6 \%$, respectively, 1 year after ALB + IVM in a subset of individuals with $>100 \mathrm{mf} / \mathrm{mL}$ at baseline. In this study, we observed reductions of $26.7 \%$ and $79.1 \%$, respectively, for these parameters in heavily infected subjects after two semiannual doses of ALB alone. The longitudinal results obtained in Seke Pembe for those subjects who were infected at baseline and tested again after ALB (a situation similar to a clinical trial) are impressive $(36.8 \% \mathrm{mf}$ clearance and $92.9 \%$ reduction in $\mathrm{mf}$ counts along with partial clearance of filarial antigenemia). They suggest that repeated MDA with ALB alone may be able to eliminate LF in communities (assuming high therapeutic coverages). The future examinations planned in Seke Pembe at 24 and 36 months (6 months after the fifth and seventh ALB MDAs) will provide additional data on this point.

Other clinical trials using ALB + IVM reported a stronger effect of this combination on $W$. bancrofti than that reported from Ghana. For example, a study from Tanzania reported that $\mathrm{mf}$ prevalence was reduced by about $80 \% 1$ year after a first treatment, ${ }^{10}$ and a study from Mali reported total $\mathrm{mf}$ clearance in $43 \%$ of the subjects treated and a decrease in $\mathrm{mf}$ densities of about $98 \% 1$ year after standard ALB + IVM treatment. ${ }^{18}$ In Sri Lanka, a 99\% reduction in $\mathrm{mf}$ densities after 1 year and $\mathrm{mf}$ clearance in $69.2 \%$ at 15 months were observed. ${ }^{24}$ However, in the Sri Lanka trial, a single dose of ALB dose alone decreased mf loads by about $93 \%$ at 1 year. Although IVM + ALB may be superior to ALB alone, ALB clearly has some activity against $W$. bancrofti, and it provides a safe alternative to IVM + ALB for MDA in central Africa.

The total clearance of CFA in some subjects and the decrease in the ICT scores observed in others suggest that two doses of ALB separated by 6 months had a partial macrofilaricidal activity against $W$. bancrofti. We assume that ICT test scores are correlated with adult worm infection intensities. Thus, it will be interesting to observe additional changes in ICT results after additional rounds of MDA.

Because STH infections were highly prevalent $(\geq 50 \%$ in school-aged children) in Seke Pembe, this community was eligible for semiannual MDA with ALB, regardless of the presence of LF in the community. ${ }^{25}$ High STH rates in the population together with the absence of basic sanitation services in Seke Pembe suggest that the environment is heavily contaminated with nematode eggs and that the force of infection is substantial. Mathematical modeling studies recently suggested that, in such settings, community MDA and more frequent treatments are more likely to achieve a significant impact (and possibly, interrupt transmission of STHs) than programs using selective MDA for school children only on an annual basis. ${ }^{26,27}$ Our preliminary results tend to support the modelers' predictions. In Seke Pembe, the first two rounds of MDA with ALB at high coverage had a significant impact on STH infection levels, with increasing effectiveness for T. trichiura, A. lumbricoides, and hookworm. 
TABLE 4

Impact of two semiannual community treatments with ALB on A. lumbricoides infection

\begin{tabular}{|c|c|c|c|c|}
\hline A. lumbricoides & 2012 & 2013 & Reduction (\%) & $P$ value \\
\hline Population aged 5-14 years old & $N=180$ & $N=121$ & & \\
\hline Prevalence (\%) & $71.1[64.4-77.8]^{*}$ & $48.8[39.7-57.8]$ & 31.4 & $<0.001$ \\
\hline GM of positive counts (epg) & $11,902.6[8,827.9-16,048.0]$ & $4,547.2[2,560.2-8,075.8]$ & 61.8 & 0.001 \\
\hline AM of positive counts (epg) & $24,926.6[20,732.6-29,120.6]$ & $13,940.8[10,260.3-17,621.2]$ & 44.1 & \\
\hline Population aged $\geq 15$ years old & $N=282$ & $N=214$ & & \\
\hline Prevalence (\%) & $47.2[41.3-53.0]$ & $29.4[23.3-35.6]$ & 37.6 & $<0.001$ \\
\hline GM of positive counts (epg) & $3,481.8[2,467.4-4,913.1]$ & $3,692.8[2,368.5-5,757.0]$ & -6.1 & 0.796 \\
\hline AM of positive counts (epg) & $10,207.4[7,804.8-12,610.0]$ & $9,010.5[6,477.9-11,543.1]$ & 11.7 & \\
\hline Total population & $N=462$ & $N=335$ & & \\
\hline Prevalence (\%) & $56.4[52.0-61.0]$ & $36.4[31.2-41.6]$ & 35.5 & $<0.001$ \\
\hline GM of positive counts (epg) & 6,362.4 [5,008.9-8,081.7] & 4,083.8 [2,862.1-5,827.8] & 35.8 & 0.012 \\
\hline AM of positive counts (epg) & $17,426.0[14,884.4-19,967.6]$ & $11,394.8[9,173.8-1,3615.8]$ & 34.6 & 0.012 \\
\hline
\end{tabular}

Although the prevalence rate for $T$. trichiura was not reduced much by ALB, MDA dramatically reduced Trichuris egg counts, and this effect was most impressive in heavily infected individuals, who were mostly children. ALB also reduced Ascaris prevalence and intensity, again with a slightly higher impact in children. However, the most impressive impact of MDA on STH infections was on hookworm. Hookworm infections were less common than Ascaris or Trichuris infections in the baseline survey, and hookworm infection intensities were low. ALB had a dramatic impact on hookworm infections; no hookworm eggs were detected in stools from children after the second round of MDA, and only a few light infections were detected after MDA in adults. The high efficacy of ALB on hookworm is well-documented, but we were surprised to see that hookworm was almost completely eliminated in this community after only two rounds of ALB. Our results suggest that semiannual MDA with ALB might be able to eliminate hookworm infections in areas with low baseline infection rates and intensities. Other studies are needed to assess the impact of community MDA in areas with higher hookworm burdens.

It is unclear whether semiannual ALB alone is less effective than combination MDA with IVM + ALB on STH infections. Several studies have shown that IVM + ALB is more effective than ALB alone for Trichuris infection but not for Ascaris or hookworm. ${ }^{28-31}$ Again, it will be interesting to follow STH infection rates and intensities at later time points in Seke Pembe, and data from this study and others should be used to ground truth and refine mathematical models for STH.

No serious adverse events were reported after two rounds of ALB MDA in Seke Pembe, but a few people reported short-term abdominal discomfort. Some individuals also reported positive effects of treatment, with feelings of relief, especially after expelling worms in their stools. These observations confirm the well-documented safety of ALB.

The WHO recommended integrated and coordinated preventive chemotherapy (PC) for human helminthiases in $2006,{ }^{25}$ and these recommendations have recently been reaffirmed in a second report on neglected tropical diseases (NTDs). ${ }^{32}$ With 33 of 40 countries requiring interventions for three or more NTDs, integrated PC is a high priority public health intervention for Africa. In Seke Pembe, after only two rounds of semiannual ALB, the extra benefits of the intervention for LF elimination are already obvious. In addition, our study confirms the feasibility of such an integrated approach, including concomitant and coordinated surveys to monitor the impact of MDA on LF and STH rates and levels in sentinel sites, which were recently reported from Benin. ${ }^{33}$ We believe that the high therapeutic coverage rate achieved in Seke Pembe was essential for the excellent results that we have observed to date. One of the main challenges ahead will be to maintain high compliance in this community and broader public health programs that provide ALB or other MDA regimens two times per year.

This is the first community study of the impact of MDA with ALB alone on LF, and we did not study the relative merits of community-directed treatment versus MDA provided by public health officials. Our study documented the effect of ALB in people who took the drug. Compliance is a major issue for all MDA programs for control or elimination of NTDs, and it is clear that ALB MDA will not work without adequate compliance.

TABLE 5

Impact of two semiannual community treatments with ALB on T. trichiura infection

\begin{tabular}{|c|c|c|c|c|}
\hline T. trichiura & 2012 & 2013 & Reduction (\%) & $P$ value \\
\hline Population aged 5-14 years old & $N=180$ & $N=121$ & & \\
\hline Prevalence $(\%)$ & $92.2[88.3-96.2]^{*}$ & $80.2[73.0-87.4]$ & 13.1 & $<0.001$ \\
\hline GM of positive counts (epg) & 897.7 [714.9-1,127.0] & $384.1[269.8-546.6]$ & 57.2 & $<0.001$ \\
\hline AM of positive counts (epg) & $2,134.8[1,634.0-2,635.7]$ & $1,367.5[769.4-1,965.6]$ & 35.9 & \\
\hline Population aged $\geq 15$ years old & $N=282$ & $N=214$ & & \\
\hline Prevalence (\%) & $69.9[64.5-75.2]$ & $65.9[59.5-72.3]$ & 5.7 & 0.357 \\
\hline GM of positive counts (epg) & $263.4[213.1-325.6]$ & $168.9[128.8-221.4]$ & 35.9 & 0.005 \\
\hline AM of positive counts (epg) & $798.2[512.2-1,084.2]$ & $804.9[244.3-1,365.6]$ & -0.8 & \\
\hline Total population & $N=462$ & $N=335$ & & \\
\hline Prevalence $(\%)$ & 78.6 [74.8-82.3] & 71.0 [66.2-75.9] & 9.8 & 0.015 \\
\hline GM of positive counts (epg) & $461.7[390.7-545.4]$ & $236.2[189.5-294.3]$ & 48.8 & $<0.001$ \\
\hline AM of positive counts (epg) & $1,409.5[1125.7-1693.2]$ & $1,034.2[623.5-1444.9]$ & 26.6 & \\
\hline
\end{tabular}

* Numbers in brackets indicate $95 \%$ CIs. 
TABLE 6

Impact of two semiannual community treatments with ALB on hookworm infection

\begin{tabular}{|c|c|c|c|c|}
\hline Hookworm & 2012 & 2013 & Reduction (\%) & $P$ value \\
\hline Population aged 5-14 years old & $N=180$ & $N=121$ & & \\
\hline Prevalence $(\%)$ & $4.4[1.4-7.5]^{*}$ & 0 & 100 & \\
\hline GM of positive counts (epg) & $22.6[10.5-47.5]$ & 0 & 100 & \\
\hline AM of positive counts (epg) & $33.0[4.2-61.8]$ & 0 & 100 & \\
\hline Population aged $\geq 15$ years old & $N=282$ & $N=214$ & & \\
\hline Prevalence $(\%)$ & $7.8[4.7-1.1]$ & $0.9[0.0-2.2]$ & 88.1 & $<0.001$ \\
\hline GM of positive counts (epg) & $50.5[33.5-76.9]$ & $17.0[0.3-1147.7]$ & 66.3 & 0.072 \\
\hline AM of positive counts (epg) & $80.2[35.0-125.4]$ & $18.0[0.0-94.2]$ & 77.6 & \\
\hline Total population & $N=462$ & $N=335$ & & \\
\hline Prevalence (\%) & $6.5[4.2-8.7]$ & $0.6[0.0-1.4]$ & 90.7 & $<0.001$ \\
\hline GM of positive counts (epg) & $40.8[28.4-58.5]$ & $17.0[0.3-1147.7]$ & 58.3 & 0.167 \\
\hline AM of positive counts (epg) & $67.6[33.7-101.5]$ & $18.0[0.0-94.2]$ & 73.4 & \\
\hline
\end{tabular}

*Numbers in brackets indicate $95 \%$ CIs.

One potential disadvantage of ALB MDA is that this regimen may lead to the more rapid development of resistance to benzimidazoles by filarial worms and STH than ALB + IVM. More time is needed to determine the number of rounds or years of MDA of ALB that will be required to interrupt LF transmission, but we suspect that this can be achieved with as few as five rounds over 24 months in areas with low to moderate baseline infection rates (assuming high compliance). The long generation time of $W$. bancrofti reduces the risk of resistance in this time frame, and in any case, IVM is not likely to reduce development of drug resistance of $W$. bancrofti to ALB because of its limited macrofilaricial activity on adult filarial worms. The resistance risk may be higher for STH. To date, ALB resistance has not been reported in human STH, but recommendations for monitoring drug efficacy for early detection of suboptimal responses have been proposed, ${ }^{34-37}$ and we think that this should be a high priority in areas that use semiannual MDA with ALB alone. The potential risk of ALB resistance in STH would favor restricting the use of ALB alone for MDA to areas where IVM cannot be safely used.

Received October 20, 2014. Accepted for publication December 9, 2014.

Published online March 9, 2015.

Acknowledgments: The authors thank the residents of Seke Pembe (especially the village chiefs and deputies) for their active participa-

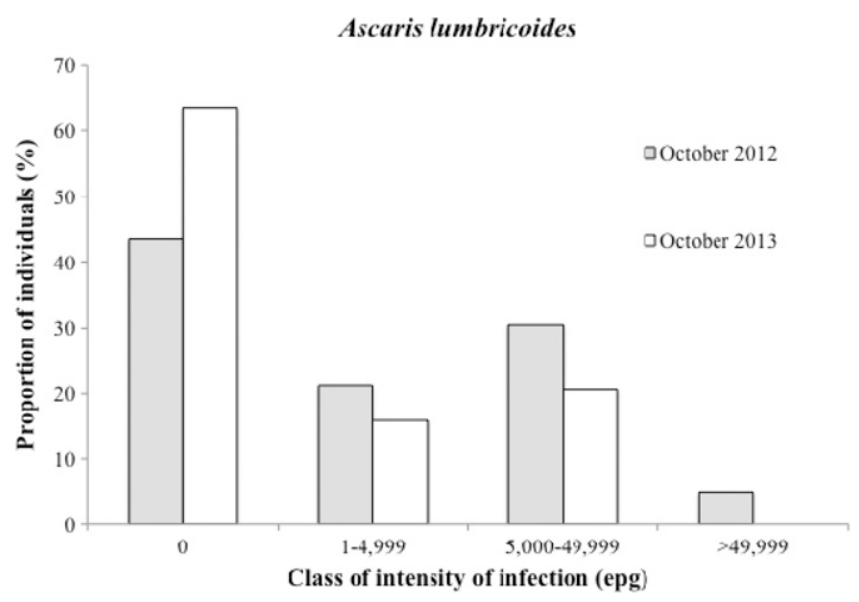

FIgURE 2. A. lumbricoides infection levels before and 6 months after two semiannual community treatments with ALB. tion in the study. We also thank the personnel from the Ministry of Health and Population, Republic of Congo for their assistance in the field (Dr. Maurice Mpompolo, Mr. Dieudonné Boungou, Mrs. Albertine Mpika, and Messrs. Valentin Angoni, Clément Biyoukala, Gyslain Boukete, Daniel Mavoungou Dibamba, Célestin Ibounaga, Sébastien Lemboumi, Anicet Madoulou, César Manene, Jean Ngoma, Magloire Ntama, and Gilbert Nzaou).

Financial support: This research was funded by Grant GH5342 from the Bill \& Melinda Gates Foundation.

Disclaimer: The funders had no role in study design, data collection and analysis, decision to publish, or preparation of the manuscript. The findings and conclusions contained within are those of the authors and do not necessarily reflect positions or policies of the Bill \& Melinda Gates Foundation.

Authors' addresses: Sébastien D. S. Pion, Cédric B. Chesnais, and Michel Boussinesq, Institut de Recherche pour le Développement, Unité Mixte Internationale 233, Montpellier, France, E-mails: sebastien.pion@ird.fr, cedric.chesnais@ird.fr, and michel.boussinesq@ ird.fr. Jean Bopda, Filariasis and Other Tropical Diseases Research Centre, Yaoundé, Cameroon, E-mail: f_bopda@yahoo.fr. Frédéric Louya and François Missamou, Programme National de Lutte contre l'Onchocercose, Direction de l'Epidémiologie et de la Lutte contre la Maladie, Ministère de la Santé et de la Population, Brazzaville, Republic of Congo, E-mails: missamou_franc@yahoo.com and fredericlouya@yahoo.fr. Peter U. Fischer and Gary J. Weil, Infectious Diseases Division, Washington University School of Medicine, St. Louis, MO, E-mails: PUfische@dom.wustl.edu and gweil@dom.wustl. edu. Andrew C. Majewski, Neglected Tropical Diseases Support Center, Task Force for Global Health, Decatur, GA, E-mail: amajewski@ taskforce.org.

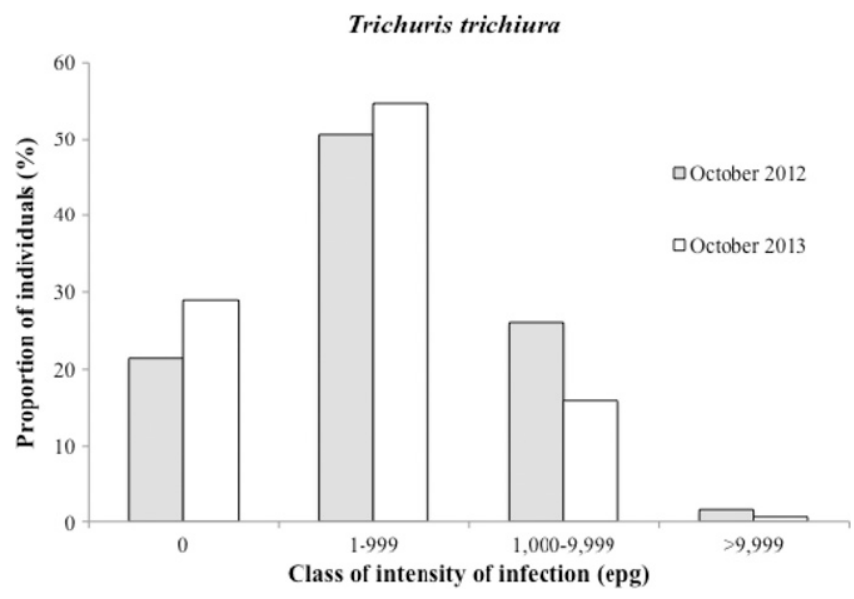

FiguRE 3. T. trichiura infection levels before and 6 months after two semiannual community treatments with ALB. 


\section{REFERENCES}

1. World Health Organization, 2012. Provisional strategy for interrupting lymphatic filariasis transmission in loiasis-endemic countries. Proceedings of the Meeting on Lymphatic Filariasis, Malaria and Integrated Vector Management; Accra, Ghana, March 5-9, 2012.

2. Ottesen EA, Hooper PJ, Bradley M, Biswas G, 2008. The global programme to eliminate lymphatic filariasis: health impact after 8 years. PLoS Negl Trop Dis 2: e317.

3. Carme B, Ebikili B, Mbitsi A, Copin N, 1991. Essai thérapeutique de l'ivermectine au cours de la loase à moyenne et forte microfilarémie. Ann Soc Belg Med Trop 71: 47-50.

4. Gardon J, Gardon-Wendel N, Demanga-Ngangue, Kamgno J, Chippaux JP, Boussinesq M, 1997. Serious reactions after mass treatment of onchocerciasis with ivermectin in an area endemic for Loa loa infection. Lancet 350: 18-22.

5. Boussinesq M, Gardon J, Gardon-Wendel N, Chippaux J-P, 2003. Clinical picture, epidemiology and outcome of Loa-associated serious adverse events related to mass ivermectin treatment of onchocerciasis in Cameroon. Filaria J 2 (Suppl 1): S4.

6. Addiss D; Global Alliance to Eliminate Lymphatic Filariasis, 2010. The 6th Meeting of the Global Alliance to Eliminate Lymphatic Filariasis: a half-time review of lymphatic filariasis elimination and its integration with the control of other neglected tropical diseases. Parasit Vectors 3: 100.

7. Bockarie MJ, Deb RM, 2010. Elimination of lymphatic filariasis: do we have the drugs to complete the job? Curr Opin Infect Dis 23: 617-620.

8. Klion AD, Massougbodji A, Horton J, Ekoue S, Lanmasso T, Ahouissou NL, Nutman TB, 1993. Albendazole in human loiasis: results of a double-blind, placebo-controlled trial. J Infect Dis 168: 202-206.

9. Ismail MM, Jayakody RL, Weil GJ, Fernando D, De Silva MS, De Silva GA, Balasooriya WK, 2001. Long-term efficacy of single-dose combinations of albendazole, ivermectin and diethylcarbamazine for the treatment of bancroftian filariasis. Trans $R$ Soc Trop Med Hyg 95: 332-335.

10. Makunde WH, Kamugisha LM, Massaga JJ, Makunde RW, Savael ZX, Akida J, Salum FM, Taylor MJ, 2003. Treatment of co-infection with bancroftian filariasis and onchocerciasis: a safety and efficacy study of albendazole with ivermectin compared to treatment of single infection with bancroftian filariasis. Filaria $J$ 2: 15

11. Wamae CN, Njenga SM, Ngugi BM, Mbui J, Njaanake HK, 2011. Evaluation of effectiveness of diethylcarbamazine/albendazole combination in reduction of Wuchereria bancrofti infection using multiple infection parameters. Acta Trop 120 (Suppl 1): S33-S38.

12. Ottesen EA, Duke BO, Karam M, Behbehani K, 1997. Strategies and tools for the control/elimination of lymphatic filariasis. Bull World Health Organ 75: 491-503.

13. Ottesen EA, 2006. Lymphatic filariasis: treatment, control and elimination. Adv Parasitol 61: 395-441.

14. Stolk WA, ten Bosch QA, de Vlas SJ, Fischer PU, Weil GJ, Goldman AS, 2013. Modeling the impact and costs of semiannual mass drug administration for accelerated elimination of lymphatic filariasis. PLoS Negl Trop Dis 7: e1984.

15. Panicker KN, Krishnamoorthy K, Sabesan S, Prathiba J, Abidha, 1991. Comparison of effects of mass annual and biannual single dose therapy with diethylcarbamazine for the control of Malayan filariasis. Southeast Asian J Trop Med Public Health 22: 402-411.

16. Cartel JL, Spiegel A, Nguyen Ngnoc L, Cardines R, Plichart R, Martin PM, Roux JF, 1991. Single versus repeated doses of ivermectin and diethylcarbamazine for the treatment of Wuchereria bancrofti var. pacifica microfilaremia. Results at 12 months of a double-blind study. Trop Med Parasitol 42: 335-338.

17. Cartel JL, Spiegel A, Nguyen Ngnoc L, Cardines R, Plichart R, Martin PM, Roux JF, Moulia-Pelat JP, 1992. Compared efficacy of repeated annual and semi-annual doses of ivermectin and diethylcarbamazine for prevention of Wuchereria bancrofti filariasis in French Polynesia. Final evaluation. Trop Med Parasitol 43: 91-94.
18. Dembele B, Coulibaly YI, Dolo H, Konate S, Coulibaly SY, Sanogo D, Soumaoro L, Coulibaly ME, Doumbia SS, Diallo AA, Traore SF, Diaman Keita A, Fay MP, Nutman TB, Klion AD, 2010. Use of high-dose, twice-yearly albendazole and ivermectin to suppress Wuchereria bancrofti microfilarial levels. Clin Infect Dis 51: 1229-1235.

19. Kazura JW, 2010. Higher-dose, more frequent treatment of Wuchereria bancrofti. Clin Infect Dis 51: 1236-1237.

20. Chesnais CB, Missamou F, Pion SDS, Bopda J, Louya F, Majewski AC, Weil GJ, Boussinesq M, 2013. Semi-quantitative scoring of an immunochromatographic test for circulating filarial antigen. Am J Trop Med Hyg 89: 916-918.

21. World Health Organization, 1998. Guidelines for the Evaluation of Soil-Transmitted Helminthiases and Schistosomiasis at Community Level. Geneva: World Health Organization.

22. Critchley J, Addiss D, Ejere H, Gamble C, Garner P, Gelband H, 2005. Albendazole for the control and elimination of lymphatic filariasis: systematic review. Trop Med Int Health 10: 818-825.

23. Dunyo SK, Nkrumah FK, Simonsen PE, 2000. Single-dose treatment of Wuchereria bancrofti infections with ivermectin and albendazole alone or in combination: evaluation of the potential for control at 12 months after treatment. Trans $R$ Soc Trop Med Hyg 94: 437-443.

24. Ismail MM, Jayakody RL, Weil GJ, Nirmalan N, Jayasinghe K, Abeyewickrema W, Sheriff MR, Rajaratnam HN, Amarasekera N, De Silva D, 1998. Efficacy of single dose combinations of albendazole, ivermectin and diethylcarbamazine for the treatment of bancroftian filariasis. Trans $R$ Soc Trop Med Hyg 92: 94-97.

25. World Health Organization, 2006. Preventive Chemotherapy in Human Helminthiasis: Coordinated Use of Anthelminthic Drugs in Control Interventions: A Manual for Health Professionals and Programme Managers. Available at: http://books.google.com/ books?hl=en \&lr=\&id=5qJvG5Eek1cC\&oi=fnd\&pg=PR4\&dq= Preventive+chemotherapy+in+human+helminthiasis+(WHO) \&ots=IdgsIVxekL\&sig=6dmMeAu2tsjZB_cTbgZSsbbCpxU. Accessed January 16, 2015.

26. Anderson R, Truscott J, Hollingsworth TD, 2014. The coverage and frequency of mass drug administration required to eliminate persistent transmission of soil-transmitted helminths. Philos Trans R Soc Lond B Biol Sci 369: 20130435.

27. Truscott JE, Hollingsworth TDI, Brooker SJ, Anderson RM, 2014. Can chemotherapy alone eliminate the transmission of soil transmitted helminths? Parasit Vectors 7: 1-8.

28. Beach MJ, Streit TG, Addiss DG, Prospere R, Roberts JM, Lammie PJ, 1999. Assessment of combined ivermectin and albendazole for treatment of intestinal helminth and Wuchereria bancrofti infections in Haitian schoolchildren. Am J Trop Med Hyg 60: 479-486.

29. Ismail MM, Jayakody RL, 1999. Efficacy of albendazole and its combinations with ivermectin or diethylcarbamazine (DEC) in the treatment of Trichuris trichiura infections in Sri Lanka. Ann Trop Med Parasitol 93: 501-504.

30. Knopp S, Mohammed KA, Speich B, Hattendorf J, Khamis IS, Khamis AN, Stothard JR, Rollinson D, Marti H, Utzinger J, 2010. Albendazole and mebendazole administered alone or in combination with ivermectin against Trichuris trichiura: a randomized controlled trial. Clin Infect Dis 51: 1420-1428.

31. Belizario VY, Amarillo ME, De Leon WU, De Los Reyes AE, Bugayong MG, Macatangay B, 2003. A comparison of the efficacy of single doses of albendazole, ivermectin, and diethylcarbamazine alone or in combinations against Ascaris and Trichuris spp. Bull World Health Organ 81: 35-42.

32. World Health Organization, 2013. Sustaining the Drive to Overcome the Global Impact of Neglected Tropical Diseases: Second WHO Report on Neglected Tropical Diseases: Summary. Geneva: World Health Organization.

33. Chu BK, Gass K, Batcho W, 'Ake M, Dorkenoo AM, Adjinacou E, Mafi', Addiss DG, 2014. Pilot assessment of soil-transmitted helminthiasis in the context of transmission assessment surveys for lymphatic filariasis in Benin and Tonga. PLoS Negl Trop Dis 8: e2708.

34. Albonico M, Engels D, Savioli L, 2004. Monitoring drug efficacy and early detection of drug resistance in human soil-transmitted 
nematodes: a pressing public health agenda for helminth control. Int J Parasitol 34: 1205-1210.

35. Vercruysse J, Albonico M, Behnke JM, Kotze AC, Prichard RK, McCarthy JS, Montresor A, Levecke B, 2011. Is anthelmintic resistance a concern for the control of human soil-transmitted helminths? Int J Parasitol Drugs Drug Resist 14: 14-27.

36. Vercruysse J, Behnke JM, Albonico M, Ame SM, Angebault C, Bethony JM, Engels D, Guillard B, Nguyen TV, Kang G, Kattula D, Kotze AC, McCarthy JS, Mekonnen Z, Montresor
A, Periago MV, Sumo L, Tchuenté LA, Dang TC, Zeynudin A, Levecke B, 2011. Assessment of the anthelmintic efficacy of albendazole in school children in seven countries where soil-transmitted helminths are endemic. PLoS Negl Trop Dis 5: e948.

37. Diawara A, Schwenkenbecher JM, Kaplan RM, Prichard RK, 2013. Molecular and biological diagnostic tests for monitoring benzimidazole resistance in human soil-transmitted helminths. Am J Trop Med Hyg 88: 1052-1061. 